\title{
Psychiatry in the Sultanate of Oman
}

\section{Hamed Al-Sinawi ${ }^{1}$ and Samir Al-Adawi ${ }^{2}$}

${ }^{1}$ Senior Registrar, Department of Behavioural Medicine, Sultan Qaboos University Hospital, Muscat, Oman, email senawi@squ.edu.om

${ }^{2}$ Lecturer, Department of Behavioural Medicine, College of Medicine and Health Sciences, Sultan Qaboos University, Muscat, Oman, email adawi@squ.edu.om

Many people with psychiatric illness are still unlikely to seek psychiatric help until they have reached an advanced stage of irreversible pathology or until 'treatment shopping' from complementary and alternative medicine has failed to provide any benefit.

$$
T_{\text {eis }}^{\text {he }}
$$
he Sultanate of Oman is located in the southeast of the Arabian Peninsula. It has a distinctive history and subcultures. Its seafaring tradition has endowed the country with various ethnic and linguistic groups, with Arabic being a dominant language and Ibadhi being the dominant sect of Islam (Al-Nami, 197I). Oman in the 1970s saw rapid development, triggered by the discovery of oil, which took place under enlightened new political leadership.

\section{Attitudes to mental illness}

As in many traditional communities, modern psychiatric services have yet to play a dominant role in the care of people with psychiatric disorders. Mental illness was largely the preserve of traditional healers, and many people with psychiatric illness are still unlikely to seek psychiatric help until they have reached an advanced stage of irreversible pathology or until 'treatment shopping' from complementary and alternative medicine has failed to provide any benefit (Al-Adawi et al, 2002a).

More positively, however, because an altered mental state is attributed to jinn (and religious teaching affirms the existence of such agents), there is little evidence of pervasive stigma towards people with mental illness in traditional Omani society (Al-Adawi et al, 2002a).

\section{Common psychiatric illness}

Although no formal epidemiological study of psychiatric illness has yet been conducted, anecdotal and impressionistic reports suggest that many types of mental disorders encountered in other countries are common in Oman, although with culturally determined differences in the types of reaction and the incidence (Al-Adawi et al, 1997, 200 I, 2002b; Chand et al, 2000).

The Omani population is undergoing a 'demographic transition', with declining death rates complemented by high birth rates. This is likely to be accompanied by an increase in the number of people with psychiatric disorders. There is also an indication that the country is bracing itself for the social and economic consequences of a more youthful population, with far more job-seekers than the labour market can absorb. The traditional passage to adulthood is also changing, as youngsters are expected to marry late and to have children when they are well into their 20s. However, the 'adolescent turmoil' seen in Western societies is not evident in Omani society, which emphasises family obedience.

Some maladjustment indicators of youth are becoming increasingly common. Firstly, the incidence of deliberate self-harm increased from 1.9 cases per 100000 in 1993 to 12.8 per 100000 by 1998 among female adolescents (Zaidan et al, 2002). Secondly, there has been an increase in the numbers of addicts seeking rehabilitation, drug overdoses and drugrelated deaths; there has also been a rise in drug shipments confiscated by law enforcement officers (Al-Harthi \& Al-Adawi, 2002). The popular mindaltering substance in Oman appears to be cannabis, although the extent of its use is hard to quantify. Finally, there is evidence that the traditional admiration of a plumpish figure is eroding, and that eating disorders are becoming increasingly common in young Omanis (Al-Adawi et al, 1999).

\section{Psychiatric services}

Psychiatry in Oman is generally based on the AngloAmerican model. The first steps towards instituting a system in Oman of biomedical care for psychiatric patients were taken in the mid-1970s in a missionary hospital, Al-Rahma Hospital, located in the capital, Muscat, in the northern part of the country. An Indian psychiatrist dispensed out-patient services, which later developed into the provision of custodial care for people with acutely disturbed behaviour. Parallel services evolved in the late 1970s in the southern part of the country.

The first specialised psychiatric hospital, Ibn Sina, was opened in 1983. It started with two wards, one for male and the other for female patients; custodial care was provided for severely disturbed patients and there were also out-patient services. The one hospital catered for the needs of the whole population (then nearly 1.5 million). In the early 1990s, a psychiatric teaching service was initiated within the teaching hospital of Sultan Qaboos University.

There is a universal free mental health service for Omani nationals. Although the majority of patients with a psychiatric illness are likely to 'somatise', individuals whose lives are compromised by psychological disorders are entitled to disability benefits, which are dispensed by the Ministry of Social Affairs. 
The World Health Organization (2005) rated the healthcare system of Oman the most efficient in the world. Despite this, as elsewhere, mental health services have been inadequately addressed. There are fewer than 15 registered mental health professionals for a population of nearly 3 million people scattered over $300000 \mathrm{~km}^{2}$. Most services are dispensed within general tertiary hospitals, but there are two specialised centres, both in the capital city. Most psychiatrists have been recruited from abroad, although some have been naturalised. Apart from the teaching hospital, the multidisciplinary infrastructures often essential for psychiatric services have remained rudimentary, owing to a lack of suitable staff. There are substandard facilities for occupational therapy and psychiatric rehabilitation remains basic.

The first point of contact for those who seek biomedical care is meant to be the primary care clinics, from where most common psychotropic medications are readily available. However, because of a lack of awareness and of suitable health education, as well as the lack of a close, informed and consistent relationship with professional care providers, there is a tendency for people to seek primary care at tertiary centres. A study has suggested that the majority of these patients present with 'unidentified medical illness' (Al Lawati et al, 2000).

To help non-specialist physicians in primary care clinics to recognise mental disorders, the Ministry of Health, the main provider of healthcare in Oman, has instituted regular training for primary health workers in the recognition of psychiatric disorders, and accompanying manuals are available to help in this endeavour.

\section{Child psychiatry}

Even though Oman's postnatal coverage is the highest among Arab nations (Sulaiman et al, 200I), with the 'baby boom' and a relatively high prevalence of consanguinity the country is also experiencing an upsurge in many childhood disorders (Kenue et al, 1995). Disorders characterised by marked regression in a variety of cognitive, motor and social skills after apparently normal development are also on the rise, with all the consequences for the parents and society of having to care for children with pervasive developmental disorders (Al-Sharbati et al, 2003).

The Ministry of Education coordinates special education and rehabilitation programmes for children with communication disorders, physical disabilities and learning disorders (Ministry of Education, 2002). The government is training school health workers to identify and intervene on behalf of vulnerable children, and some teachers are taking an active part in this campaign. As diverse organisations and professionals provide care for children, there is a need both to standardise taxonomy and to enhance crossdisciplinary communication. To this end, specialised centres for the behavioural and emotional problems of children will be essential.

\section{Policy and legislation}

Mental health policies and legislation have been forthcoming in recent years, with a decree to promulgate a substance misuse policy and a law on the control of narcotics and psychotropic medication. Furthermore, a national mental health programme has been established (World Health Organization, 2005).

\section{Education}

\section{Undergraduate education}

The duration of general medical training in students at Sultan Qaboos University is 7 years (4 years preclinical, 3 years clinical). Graduates then spend I year on internship, rotating between three out of four specialties (internal medicine, general surgery, child health, obstetrics and gynaecology).

Behavioural science, which encompasses diverse disciplines, including sociology, anthropology and psychology, is taught in the second and third years of the pre-clinical course, in order for Omani trainees to appreciate the social and cultural aspects of illness and well-being. In their sixth year, clinical students spend 8 weeks on a clinical clerkship in psychiatry, which is their first exposure to psychiatry. The 8-week programme includes supplementary lectures on various aspects of clinical psychiatry and allied fields, patient case presentation, case histories and clinical interview.

\section{Postgraduate education}

The psychiatry residency programme was introduced in 1999 under the auspices of the Oman Medical Board, which oversees postgraduate training. The average duration of the training is 4 years, during which the trainee will complete different modules each year before taking the membership examination relevant to his or her specialty.

The psychiatry residency programme did not attract applicants until recently. Five Omani doctors have now completed their residency training in psychiatry (two of whom completed their training in the UK and obtained the MRCPsych). In 2004 two new graduates started their psychiatry residency, followed by three more in 2005. Two more have enrolled in an institution of higher education in the UK, to pursue academic programmes in substance misuse and child and adolescent psychiatry.

\section{Challenges to psychiatry}

Traditional value systems in Oman are collectivist in orientation. In communal society, from birth children are brought up in an environment that ushers them into the collective mind-set; the development of selfhood is generally discouraged, as is expression of emotions. Depending on the level of education, difficulties are attributed to jinn and distress is communicated in psychosomatic rather than psychological ways (AlAdawi et al, 2002a). The fact that traditional Omanis
Because of a lack of awareness and of suitable health education, as well as the lack of a close, informed and consistent relationship with professional care providers, there is a tendency for people to seek primary care at tertiary centres.

In communal society, from birth children are brought up in an environment that ushers them into the collective mind-set; the development of self-hood

is generally discouraged, as is expression of emotions. 
attribute ill-health to external agents has two particular implications for psychiatry. When a social impropriety occurs, an individual is likely to attribute his or her difficulty to external forces like jinn, the 'evil eye' or witchcraft. It is not surprising, therefore, that many psychiatric problems are first brought to the attention of traditional healers. The second implication is that distress in Oman is not perceived in psychiatric parlance, as intra-psychic conflict. A psychiatric attempt to

With

modernisation,

the social

status of Omani

individuals has

undergone some

notable changes.

These have been

brought about by

unprecedented

prosperity,

expansion of

educational

opportunities and, most importantly

from the point of view of psychiatry, an increased

preference for an individualistic rather than

the traditional collectivistic mindset and social behaviour. heal the 'self' is always going to be difficult in a society where development of the self is not lauded.

A survey of Oman's trainees' interest in psychiatry (Al-Adawi et al, 2006) has suggested that the attitudes towards psychiatry and psychiatric services appear to be positive. However, there is little interest in psychiatry as a career. Within the context of increasing mental health problems, this is likely to represent a challenge for the country. At the moment, overseas psychiatrists are filling the gap. However, expatriate health personnel may not be well versed in local traditions and languages.

Unless the profession institutes mechanisms to decode local idioms of distress rather than adhering to biomedical models of mental illness (Littlewood \& Lipsedge, 1997), psychiatry is likely to be perceived as medicine for 'crazy people'.

Despite these caveats, within the last two decades, rapid acculturation has occurred in Oman. With modernisation, the social status of Omani individuals has undergone some notable changes. These have been brought about by unprecedented prosperity, expansion of educational opportunities and, most importantly from the point of view of psychiatry, an increased preference for an individualistic rather than the traditional collectivistic mind-set and social behaviour. With these expected changes, psychiatry will have fertile ground on which to flourish.

\section{References}

Al-Adawi, S., Burjorjee, R. N. \& Al-Issa, I. (1997) Mu Ghayeb: a culture-specific response to bereavement in Oman. International Journal of Social Psychiatry, 43, |44- I5I.

Al-Adawi, S., Ghassany, H., Al-Naamani, A., et al (1999) Sub-clinical eating disorders: preliminary study of students in Muscat. Oman Medical Journal, I5, 29-29.

Al-Adawi, S., Salmi, A., Martin, R. G., et al (200I) Zar: group distress and healing. Mental Health, Religion and Culture, 4, 47-61.

Al-Adawi, S., Dorvlo, A. S. S., Al-Ismaily, S. S., et al (2002a) Perception of and attitude towards mental illness in Oman. International Journal of Social Psychiatry, 48, 305-317.

Al-Adawi, S., Dorvlo, A. S., Burke, D. T., et al (2002b) A survey of anorexia nervosa using the Arabic version of the EAT-26 and 'gold standard' interviews among Omani adolescents. Eating and Weight Disorders, 7, 304-311.

Al-Adawi, S., Dorvlo, A. S. S., Bhaya, C., et al (2006) Withering before the sowing? A survey of Oman's tomorrow's doctors' interest in psychiatry. Education for Health (in press).

Al-Harthi, A. \& Al-Adawi, S. (2002) Enemy within? The silent epidemic of substance dependency in GCC countries. SQU journal for Scientific Research: Medical Sciences, 4, I-7.

Al Lawati, J., Al Lawati, N., Al Siddiqui, M., et al (2000) Psychological morbidity in primary healthcare in Oman: a preliminary study. Journal for Scientific Research: Medical Sciences, 2, I05110

Al-Nami, A. K. (197I) Studies in Ibadhism. Cambridge: Cambridge University Press.

Al-Sharbati, M. M., Al-Hussaini, A. A. \& Antony, S. X. (2003) Profile of child and adolescent psychiatry in Oman. Saudi Medical Journal, 24, 391-395.

Chand, S. P., Al-Hussaini, A. A., Martin, R., et al (2000) Dissociative disorders in the Sultanate of Oman. Acta Psychiatrica Scandinavica, 102, 185-187.

Kenue, R. K., Raj, A. K., Harris, P. F., et al (1995) Cytogenetic analysis of children suspected of chromosomal abnormalities. Journal of Tropical Pediatrics, 4I, 77-80.

Littlewood, R. \& Lipsedge, M. (1997) Aliens and Alienists: Ethnic Minorities and Psychiatry (3rd edn). London: Routledge.

Ministry of Education (2002) Evolution of Educational Statistics in the Sultanate, 1970-2001. Muscat: Ministry of Education.

Sulaiman, A. J., Al-Riyami, A., Farid, S., et al (200I) Oman family health survey 1995. Journal of Tropical Pediatrics, 47 (suppl I.) I-33.

World Health Organization (2005) Mental Health Atlas. Geneva: $\mathrm{WHO}$.

Zaidan, Z. A., Burke, D. T., Dorvlo, A. S., et al (2002) Deliberate self-poisoning in Oman. Tropical Medicine and International Health, 7, 549-556.

\section{Psychiatry in Qatar}

\section{Suhaila Ghuloum ${ }^{1}$ and Mohammed A. Ibrahim²}

${ }^{1}$ Consultant Psychiatrist, Psychiatry Department, Hamad Medical Corporation, PO Box 3050, Doha, Qatar, email sghuloum@hmc.org.qa

${ }^{2}$ Chairman, Psychiatry Department, Hamad Medical Corporation, email psychiatry@hmc.org.qa

\section{T}

he State of Qatar is a peninsula overlooking the Arabian Gulf, with an area of II $400 \mathrm{~km}^{2}$. The Al Thani family has ruled the country since the mid-1800s. The population of just over 860000 is of a multi-ethnic nature, and predominantly resides in the capital, Doha. Only about 20\% of the population is Qatari. Around 73\% of the population are between the ages of 15 and 64 years. Life expectancy at birth is 74.8 years for males and 73.8 years for females. The literacy rate is $94.9 \%$ for men and $82.3 \%$ for women. Arabic is the official language and English is a common second language. The economy is dominated by oil and natural gas, and the country has one of the highest per capita incomes in the world. The per capita government expenditure on health is $\$ 574$ (international dollars), which is among the highest in the region. 